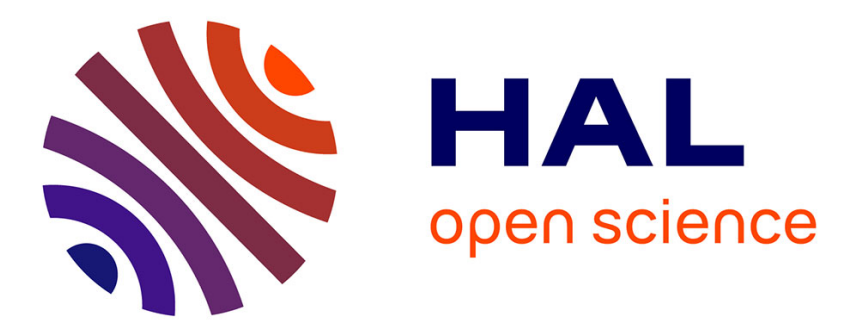

\title{
DVH-Based Inverse Planning Using Monte Carlo Dosimetry for LDR Prostate Brachytherapy
}

Konstantinos Mountris, Dimitris Visvikis, Julien Bert

\section{To cite this version:}

Konstantinos Mountris, Dimitris Visvikis, Julien Bert. DVH-Based Inverse Planning Using Monte Carlo Dosimetry for LDR Prostate Brachytherapy. International Journal of Radiation Oncology, Biology, Physics, 2018, 103 (2), pp.503-510. 10.1016/j.ijrobp.2018.09.041 . hal-02944152

\section{HAL Id: hal-02944152 \\ https://hal.science/hal-02944152}

Submitted on 21 Oct 2021

HAL is a multi-disciplinary open access archive for the deposit and dissemination of scientific research documents, whether they are published or not. The documents may come from teaching and research institutions in France or abroad, or from public or private research centers.
L'archive ouverte pluridisciplinaire HAL, est destinée au dépôt et à la diffusion de documents scientifiques de niveau recherche, publiés ou non, émanant des établissements d'enseignement et de recherche français ou étrangers, des laboratoires publics ou privés.

\section{다)(1) $(5$}

Distributed under a Creative Commons Attribution - NonCommerciall 4.0 International 


\section{Physics Contribution}

\section{DVH-Based Inverse Planning Using Monte Carlo Dosimetry for LDR Prostate Brachytherapy}

\section{Konstantinos A. Mountris, PhD, Dimitris Visvikis, PhD, and Julien Bert, PhD}

\section{LaTIM, INSERM, UMR 1101, Brest, France}

Received May 7, 2018. Accepted for publication Sep 28, 2018.

\author{
Summary \\ We proposed an inverse \\ planning method based on \\ dose-volume histogram opti- \\ mization and Monte Carlo \\ dosimetry. We compared \\ plans calculated using our \\ proposed method with clin- \\ ical plans calculated using \\ the TG-43 dosimetry \\ formalism. We demonstrated \\ that our method can provide \\ plans with higher prostate \\ dose homogeneity (up to \\ $6.1 \%$ ) and lower urethral \\ dose (up to $4.0 \%$ ) compared \\ with the clinical plans. The \\ computational time \\ $(37.5 \pm 3.2 \mathrm{~s})$ complies with \\ intraoperative time \\ restrictions.
}

\begin{abstract}
Purpose: Inverse planning is an integral part of modern low-dose-rate brachytherapy. Current clinical planning systems do not exploit the total dose information and largely use the American Association of Physicists in Medicine TG-43 dosimetry formalism to ensure clinically acceptable planning times. Thus, suboptimal plans may be derived as a result of TG-43-related dose overestimation and nonconformity with dose distribution requirements. The purpose of this study was to propose an inverse planning approach that can improve planning quality by combining dose-volume information and precision without compromising the overall execution times.

Methods and Materials: The dose map was generated by accumulating precomputed Monte Carlo (MC) dose kernels for each candidate source implantation site. The MC computational burden was reduced by using graphics processing unit acceleration, allowing accurate dosimetry calculations to be performed in the intraoperative environment. The proposed dose-volume histogram (DVH) fast simulated annealing optimization algorithm was evaluated using clinical plans that were delivered to 18 patients who underwent low-dose-rate prostate brachytherapy.

Results: Our method generated plans in $37.5 \pm 3.2$ seconds with similar prostate dose coverage, improved prostate dose homogeneity of up to $6.1 \%$, and lower dose to the urethra of up to $4.0 \%$.

Conclusions: A DVH-based optimization algorithm using MC dosimetry was developed. The inclusion of the DVH requirements allowed for increased control over the optimization outcome. The optimal plan's quality was further improved by considering tissue heterogeneity. (C) 2018 Elsevier Inc. All rights reserved.
\end{abstract}

Reprint requests to: Konstantinos A. Mountris, PhD, LaTIM, INSERM, UMR 1101, Faculté de Médecine, IBRBS 22, Av. Camille Desmoulins, 29200 Brest, France. Tel: + 33 (0)2 $98018130,+33$ (0)2 980181 30; E-mail: konstantinos.mountris@gmail.com
This work was funded by the French National Research Agency through the FOCUS project. (ANR-16-CE19-0011) and within the Investissements d'Avenir program (LabEx CAMI) under reference ANR-11LABX-0004 (Integrated project CAPRI).

Conflict of interest: none. 


\section{Introduction}

Since its introduction in 1983 by Holm et al, ${ }^{1}$ transrectal ultrasound-guided prostate brachytherapy has become a widely used treatment option for prostate cancer. Its 2 main variants are permanent low-dose-rate (LDR) ${ }^{2}$ and temporary high-dose-rate (HDR) ${ }^{3}$ brachytherapy. Even though both LDR and HDR techniques achieve similar tumor control rates, ${ }^{4}$ HDR brachytherapy allows for increased dosimetric control during treatment delivery. ${ }^{5}$

However, LDR brachytherapy remains the method of choice in many clinical centers worldwide, mainly for logistical reasons (it is a 1-time procedure). As such, dosimetry adjustment is not feasible after the end of the seed implantation procedure. Therefore, the outcome can only be ensured by precise delivery of the seeds at the planned implantation locations. However, this procedure is an arduous task that requires high experience and intuition considering the large number of candidate implantation sites.

To facilitate the treatment procedure, inverse planning has become an inseparable part of the modern LDR brachytherapy practice. The selection of the most suitable seed positions is translated into an optimization problem. The treatment's dosimetric objectives are combined in a cost function to be minimized. Metaheuristic optimization algorithms such as simulated annealing ${ }^{6}$ (SA) and genetic algorithms ${ }^{7}$ have been used to solve this optimization problem. SA and its variants (eg, fast simulated annealing [FSA]) is efficient and is the most frequently used algorithm in clinical practice. ${ }^{6,8-11}$ In the work of Lessard and Pouliot, ${ }^{11}$ treatment planning optimization of HDR prostate brachytherapy, using FSA, was performed in 41 seconds on a Pentium III $700 \mathrm{MHz}$. Recently, an alternative optimization method using a compressed sensing inspired solver for interactive treatment planning, which needed $542 \times$ less time compared with standard SA, was introduced. ${ }^{12}$

Regardless of the computational performance of the aforementioned methods, the cost function evaluation is performed on sampled points at the surface and volume of the target (prostate) and critical organs (urethra and rectum). Improper selection of these points can affect the dose distribution in the target and critical organs, ${ }^{13}$ leading to suboptimal plans. In practice, the plan's quality is evaluated based on dose-volume histograms (DVHs) for the organs of interest. The DVH should satisfy specific guidelines, such as the recommendations provided in the American Association of Physicists in Medicine (AAPM) Task Group (TG)-137 report. ${ }^{14}$ DVH-based optimization algorithms have been successfully used in clinical practice for intensity modulated radiation therapy ${ }^{15,16}$ and have been proposed for use in cervical cancer ${ }^{17}$ and prostate cancer ${ }^{18}$ brachytherapy. Although DVH-based optimization methods allow for direct control of the dosimetric objectives, they have not been widely used in clinical practice.
Furthermore, current clinical inverse planning systems for prostate brachytherapy use the AAPM TG-43 dose calculation formalism. ${ }^{19}$ This formalism permits calculation times that are acceptable for intraoperative planning. However, the TG-43 formalism overestimates the dose deposited in the prostate and the critical organs ${ }^{20-22}$ because tissue heterogeneity and interseed scattering and attenuation (ISA) are not considered. Although dosimetry based on Monte Carlo (MC) simulations can reduce these inaccuracies, the long computational times limit the use of MC simulation techniques for intraoperative dosimetry calculations. Acuros, ${ }^{23}$ a grid-based Boltzmann solver, was originally introduced as a compromise between the TG-43 dosimetry formalism and MC simulations.

Another solution for intraoperative use of MC dosimetry has been proposed by D'Amours et al. ${ }^{8} \mathrm{MC}$ simulations were introduced in HDR breast brachytherapy inverse planning. An MC dose kernel (MCDK) was precomputed for each possible dwell position and subsequently used during optimization. The optimized total dose was modeled by summing the dose contributions of the MCDKs. The computation of the MCDKs required a total time of 78 hours using a small computer cluster. Currently, MC computational times can be significantly decreased by implementing graphics processing units (GPUs). Dedicated GPU-accelerated MC codes for medical applications have already been proposed. ${ }^{24-26}$

In this work, we propose an inverse planning algorithm that combines DVH-based FSA optimization and GPUaccelerated MC dosimetry using the GGEMS (GPU Geant4-based Monte Carlo Simulation) platform. ${ }^{26,27}$ Our purpose is to contribute to the state of the art of inverse planning for LDR prostate brachytherapy. The proposed methodology provides fast and efficient optimization with improved dosimetric precision.

\section{Methods and Materials}

\section{Planning process}

Instead of the clinically used AAPM TG-43 dosimetry formalism, MC dosimetry methods were used in this work to account for the effect of tissue heterogeneities on the dose deposition. During the planning process, a computational phantom was generated from the segmentation of the patient's intraoperative ultrasound image (a detailed description is provided later). Next, the needle trajectories that penetrated the prostate without crossing any of the critical organs (urethra and rectum) were identified as possible candidates for seed placement. An MCDK was generated for each candidate implantation site using the GGEMS platform.

During inverse planning, the implant was optimized by minimizing a DVH-based cost function using FSA. ${ }^{6}$ The optimization started with a randomly selected initial plan. 
The dose map was constructed by summing the dose distribution from the precomputed MCDKs that correspond to the selected seeds. DVH metrics for cost evaluation were extracted from the final dose map. The number of seeds used for treatment was calculated using the empirical formula (see Eq. 1) introduced by Pouliot et al. ${ }^{6}$ Mean seed strength was $0.654 \mathrm{U}(0.558-0.773 \mathrm{U})$.

$$
N_{\text {seeds }}=4+\left[\left(4.674 \times V^{0.562}\right) / A\right]
$$

where $V$ represents the prostate's volume given in cubic centimeters, and $A$ is the seed strength expressed in units of air-kerma strength (U). The number 4 in Eq. 1 corresponds to "virtual" seeds with no dose contribution. The purpose of the "virtual" seeds was to allow reduction of the number of delivered seeds, if necessary, during optimization. During each iteration of the FSA, the quality of the plan was improved by single-seed swapping between randomly selected occupied and nonoccupied implantation sites.

\section{Computational phantom generation}

AAPM TG-186 reported extensively ${ }^{28}$ on the potential effects of tissue heterogeneities on the dose distribution. Especially in LDR brachytherapy, in which the photoelectric phenomenon is the dominant process, the difference in mass-energy absorption coefficients between tissue and water may result in clinically relevant dose alteration. ${ }^{20-22}$

According to TG-186 guidelines, mass-energy absorption coefficients should be derived on a voxel-by-voxel basis from computed tomography (CT) imaging. However, CT imaging is not part of the standard LDR brachytherapy workflow. Moreover, CT artifacts (eg, artifacts from metallic prosthesis) may reduce the dosimetric accuracy, and filtering techniques should be applied. ${ }^{29}$ Double energy and spectral CT have demonstrated superior tissue segmentation ability compared with single energy $\mathrm{CT}^{30}$ and may improve the dosimetric accuracy.

In this work, the patient's segmentation image (available from intraoperative contouring) was converted into a heterogeneous computational phantom that included 4 materials (prostate, urethra, rectum, and surrounding tissue). The composition of the materials was assigned following the recommendations of the International Commission on Radiation Units and Measurements Report No. $46^{31}$ and the
International Commission on Radiological Protection Publication No. 89. ${ }^{32}$ The material composition was expressed in terms of elemental mass and mass density. A detailed description of the material compositions is given in Table 1.

\section{MCDK computation}

Using GGEMS to perform GPU-parallelized MC simulations, the computational time was reduced by several orders of magnitude compared with standard MC. ${ }^{26}$ The tracklength estimator ${ }^{33}$ was used during dose recording. To achieve plan dose maps with $\approx 2 \%$ statistical uncertainty, $5 \times 10^{6}$ simulated particles were required. The simulation of dose maps with (206-279) $\times(216-231) \times(24-35)$ voxel resolution and $0.31 \times 0.30 \times 2.00 \mathrm{~mm}^{3}$ voxel size was performed in $2.0 \pm 0.5$ seconds on an NVIDIA GTX TITAN X GPU using double precision. Such computational time is in good agreement with alternative GPU-enabled dose engines. ${ }^{34}$

Despite the fast dose map generation, the plan's dose map requires several thousand modifications during inverse planning. Thus, to avoid computational overhead, we decoupled the dose calculation from inverse planning. To do so, we precomputed MCDKs for each seed that could be delivered from the available needle trajectories. During MCDK generation, the seed's geometric specifications were considered. Throughout this study, the STM1251 seed model was used. The STM1251 seed was modeled by an MC-generated phasespace, which was computed in previous work according to its geometric specifications. ${ }^{35}$ Emitted particles were recorded accounting for particle interactions inside the seed's body.

The plan's total dose map was generated accumulating $N$ MCDKs, where $N$ is the number of the seeds in the plan (see Eq. 1). This allowed relaxation of the statistical uncertainty requirement for each MCDK. Using $5 \times 10^{6} / \mathrm{N}$ simulated particles per MCDK was adequate to achieve $\approx 2 \%$ statistical uncertainty in the total dose map. Therefore, the computational time for each MCDK was reduced from $2.0 \pm 0.5$ seconds to $40 \pm 10$ milliseconds. Finally, the precomputed MCDKs were compressed by discarding the dose values recorded on the surrounding tissue because

Table 1 Material composition of the computational phantom's segmented tissues

\begin{tabular}{|c|c|c|c|c|c|c|c|c|c|c|c|c|c|}
\hline \multirow[b]{2}{*}{ Tissue } & \multicolumn{12}{|c|}{ Elemental composition ( $\%$ by mass) } & \multirow[b]{2}{*}{ Density $\left(\mathrm{g} / \mathrm{cm}^{3}\right)$} \\
\hline & $\mathrm{H}$ & $\mathrm{C}$ & $\mathrm{N}$ & $\mathrm{O}$ & $\mathrm{Na}$ & $\mathrm{Mg}$ & $\mathrm{P}$ & $\mathrm{S}$ & $\mathrm{Cl}$ & $\mathrm{K}$ & $\mathrm{Ca}$ & $\mathrm{Zn}$ & \\
\hline Prostate* & 9.76 & 9.11 & 2.47 & 78.10 & 0.21 & 0.02 & 0.10 & - & - & 0.20 & 0.02 & 0.01 & 1.045 \\
\hline Rectum $^{\dagger}$ & 10.60 & 11.50 & 2.20 & 75.10 & 0.10 & - & 0.10 & 0.10 & 0.20 & 0.10 & - & - & 0.932 \\
\hline Surrounding ${ }^{\dagger, \ddagger}$ & 10.45 & 23.22 & 2.49 & 63.02 & 0.11 & 0.01 & 0.14 & 0.20 & 0.14 & 0.20 & 0.02 & - & 1.000 \\
\hline
\end{tabular}


inverse planning was performed evaluating only the dose delivered to the prostate and critical organs. This permitted further reduction in the required processing time of MCDKs for DVH metric extraction.

\section{DVH-based FSA}

Inverse planning was performed using FSA to minimize a DVH-based cost function based on the AAPM TG-137 guidelines ${ }^{14}$ given by Eq. 2

$$
\begin{aligned}
C F & =w \Theta\left(V_{100_{L B}}-V_{100}\right) \cdot\left(V_{100_{L B}}-V_{100}\right) \\
& +\sum_{i} w \Theta\left(V_{i}-V_{i_{H B}}\right) \cdot\left(V_{i}-V_{i_{H B}}\right) \\
& +\sum_{j} w \Theta\left(D_{j}-D_{j_{H B}}\right) \cdot\left(D_{j}-D_{j_{H B}}\right)+w N_{\text {needles }},
\end{aligned}
$$

where $V_{100}, V_{i}$, and $D_{j}$ represent the DVH metric values of the plan. $V_{\text {lOoLB }}$ defines the lower limit for the prostate's $V_{100}$ metric and $V_{i H B}$ the higher limits for the prostate's $V_{i}$ metrics with $i=\{150,200\}$. Similarly, $D_{j H B}$ represents the higher limits for the $D_{j}$ metrics of the urethra with $j=\{10,30\}$ and the rectum with $j=\{2 \mathrm{cc}$, $0.1 \mathrm{cc}\}$. The weight factor $w$ controls the contribution of each objective to the cost function. The 8 objectives were considered equally significant. Therefore, $w=1 / 8$ was selected. Finally, $N_{\text {needles }}$ is the number of the needles selected to deliver the plan's seeds, and $\Theta(X)$ is the Heaviside step function operator. $\Theta(X)$ was used to penalize objectives that did not satisfy the specified DVH requirements.

During FSA, an initial plan was selected randomly and was iteratively updated by single-seed swapping. In each update, the differential DVH with bin size of 1 Gy was derived by processing the plan's compressed dose map once and was converted to cumulative DVH for the prostate, urethra, and rectum. The plan's DVH metrics were extracted from the cumulative $\mathrm{DVH}$, and the cost was subsequently evaluated. An implant update resulting in a lower cost value $(E)$ compared with the previous cost value was accepted as the current optimal seed distribution. Otherwise its acceptance probability was given by Eq. 3:

$$
P(\Delta E)=\exp (-\Delta E / T(k))
$$

where $k$ is the iteration number, and $\Delta E$ is the cost function value difference between the $k$ and $k-1$ iterations. In each iteration $k$, the annealing temperature $T(k)$ was decreased with cooling rate $(C R)$ as given by the annealing schedule described by Eq. 4:

$$
T(k)=T(k-1) \times(1-C R),
$$

where $T(k)$ is the annealing temperature at the $k^{\text {th }}$ iteration, and $C R$ receives a user-defined value in the range $[0,1]$. The selected annealing schedule ensures that the optimization algorithm always terminates.

\section{Inverse planning quality validation}

The planning quality of the proposed method (DVH-FSA) was evaluated on a retrospective clinical cohort study. Planning data of a population of 18 randomly selected patients who underwent LDR prostate brachytherapy were extracted from the commercial inverse planning system's database. All patients received treatment during the year 2016. DVH-FSA and clinical plans were compared to measure the expected performance of the proposed algorithm in a clinical setting. The clinical plans were calculated using the commercial inverse planning system VariSeed v9.0 (Varian Medical Systems, Palo Alto, CA) (using the default surface optimization module). The quality of the clinical and the DVH-FSA plans was compared regarding diversion from the AAPM TG-137 report's guidelines. To eliminate dosimetric differences resulting from statistical uncertainty, the dose maps of the clinical plans were recalculated using GGEMS using $5 \times 10^{6}$ simulated particles per clinical plan dose map.

Two simulation scenarios were considered for the clinical plans. In the first scenario (Clinical TG-43), the composition of water was assigned to all of the computational phantom's voxels. In the second scenario (Clinical $\mathrm{MC})$, the heterogeneous material composition of the prostate, critical organs, and surrounding tissue was considered (Table 1) to investigate the effect of heterogeneity on the dose map of the clinical plans. The same material composition was also used in the DVH-FSA planning.

\section{Results}

\section{Dose calculation precision}

Considering the 18 patients of this study, a mean number of $64 \pm 5$ seeds was planned to be delivered using Eq. 1. For this number of seeds, the individual MCDKs were generated with $5 \times 10^{6} / 64=78125$ simulated particles. The low number of simulated particles allowed us to compute the dose contribution for all candidate seeds (400-600 seeds) in $21.5 \pm 2.5$ seconds complying with the intraoperative planning time restrictions.

Moreover, the accumulation of the individual MCDKs resulted in total dose maps with $2.29 \% \pm 0.15 \%$ mean statistical uncertainty in the prostate. When MC simulations were performed considering the total seeds' configuration and recording $5 \times 10^{6}$ simulated particles, the mean statistical uncertainty was $2.27 \% \pm 0.16 \%$. Furthermore, reducing the mean statistical uncertainty did not notably influence the accuracy of the calculated dose distribution. When dose maps were generated with $10^{7}$ simulated particles $(\approx 1.00 \%$ mean statistical uncertainty), the average relative difference of the DVH metric values was $0.30 \%$.

\section{Treatment plan optimization}

The selected linear annealing schedule (see Eq. 4) resulted in a fixed number of iterations ( $k$ ) for the plan's 


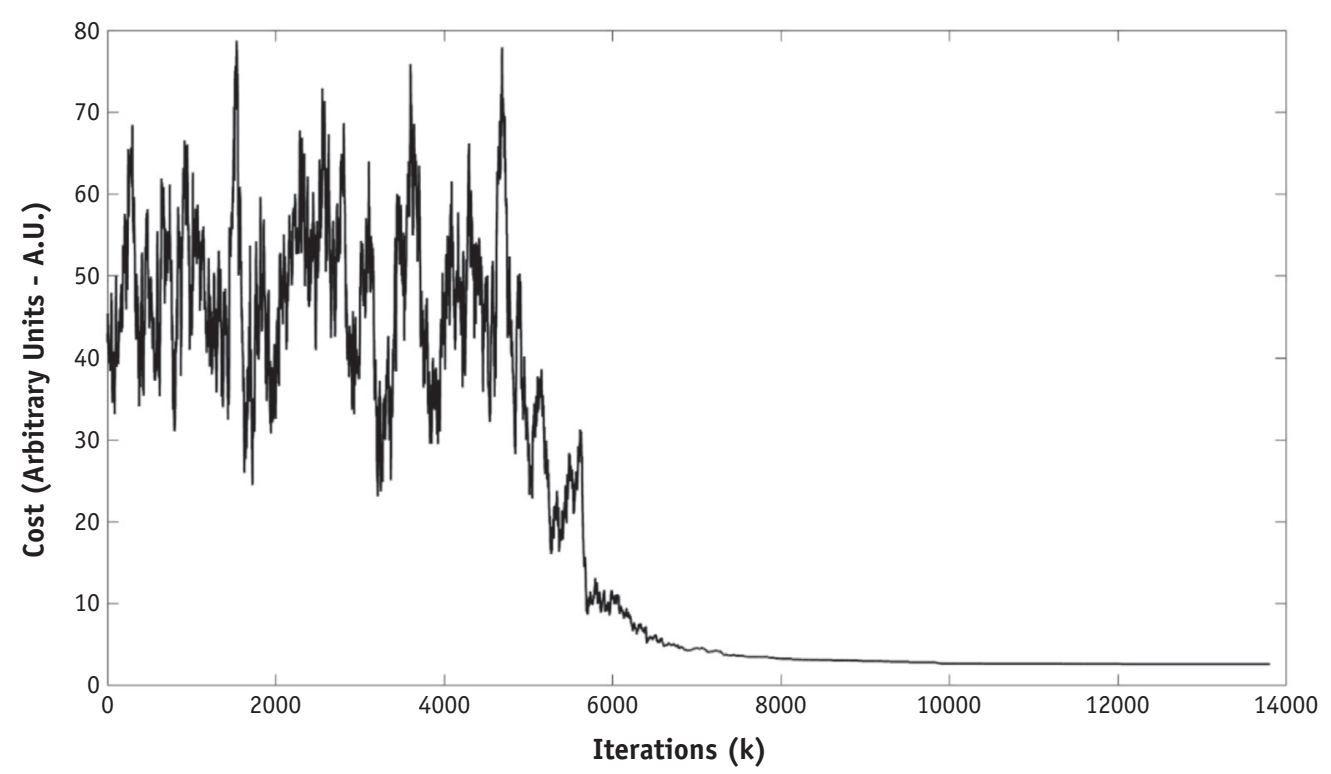

Fig. 1. Dose-volume histogram fast simulated annealing cost function minimization history. The global minimum value $\left(\right.$ Cost $=0.125 \times \mathrm{N}_{\text {needles }}$ A.U.) was satisfied at the optimization's termination (13,802 iterations).

optimization and was found to depend on the selected annealing temperature $(T)$ and cooling ratio $(C R)$ values. After evaluating several values, $T=10^{5}$ degrees and $\mathrm{CR}=0.20 \%$ were selected for this study as a good compromise between speed and convergence. The selected $T$ and $C R$ allowed a global minimum cost value in 13,802 iterations (see Fig. 1) and $16.0 \pm 2.0$ seconds (on a CPU Intel Core i7 4720HQ). In combination with the required computational time of $21.5 \pm 2.5$ seconds for the precomputation of the MCDKs, the total treatment plan computational time was $37.5 \pm 3.2$ seconds.

The DVH-FSA plans satisfied the AAPM TG-137 planning recommendations for all 18 patients with improved quality compared with the Clinical TG-43 plans. The prostate $\mathrm{V}_{150}$ dose metric was improved by $6.1 \%$, and the urethra $\mathrm{D}_{10}$ metric was improved by $4.0 \%$. No significant difference was observed for the prostate $V_{100}$ and rectum $D_{2 c c}$ metrics. Only the rectum $D_{0.1 c c}$ metric was of inferior quality compared with Clinical TG-43 plans, with a mean relative difference of $6.4 \%$.

Because of the dose overestimation associated with the AAPM TG-43 dose formalism, the Clinical TG-43 implant quality does not represent the actual deposited dose. Comparing the Clinical TG-43 with the Clinical MC plans, wherein tissue heterogeneity was considered, the dose overestimation of the former in the prostate and critical organs was demonstrated. The Clinical TG-43 plans overestimated the prostate $\mathrm{V}_{100}$ by up to $2.2 \%$.

The planning quality of the Clinical TG-43, Clinical MC, and DVH-FSA plans is given in Table 2 in terms of

Table 2 DVH metrics comparison between the investigated scenarios

\begin{tabular}{|c|c|c|c|c|c|c|}
\hline Tissue & DVH metric & $\begin{array}{c}\text { Clinical } \\
\text { TG-43 }\end{array}$ & Clinical MC & $\begin{array}{c}\text { MC vs TG-43 R } \\
\text { Diff }(\%)\end{array}$ & DVH-FSA & $\begin{array}{c}\text { DVH-FSA vs } \\
\text { TG-43 R Diff }(\%)\end{array}$ \\
\hline \multirow[t]{3}{*}{ Prostate } & $\mathrm{V}_{100}(\%)$ & $96.8 \pm 1.5$ & $94.7 \pm 2.3$ & -2.2 & $96.6 \pm 1.0$ & -0.2 \\
\hline & $\mathrm{V}_{200}(\%)$ & $20.7 \pm 2.2$ & $18.9 \pm 2.5$ & -8.7 & $19.6 \pm 0.5$ & -5.3 \\
\hline & $\mathrm{D}_{90}(\mathrm{~Gy})$ & $163.4 \pm 4.9$ & $156.7 \pm 6.4$ & -4.3 & $162.4 \pm 3.8$ & -0.6 \\
\hline Urethra & $\mathrm{D}_{10}(\mathrm{~Gy})$ & $184.6 \pm 8.5$ & $172.7 \pm 8.9$ & -6.5 & $177.3 \pm 11.8$ & -4.0 \\
\hline Rectum & $\mathrm{D}_{0.1 \mathrm{cc}}(\mathrm{Gy})$ & $156.6 \pm 14.8$ & $153.6 \pm 15.7$ & -1.9 & $166.7 \pm 21.2$ & +6.4 \\
\hline Implantation device & $\begin{array}{l}\text { Clinical } \\
\text { TG-43 }\end{array}$ & Clinical MC & $\begin{array}{c}\text { MC vs TG-43 A } \\
\text { Diff }(\%)\end{array}$ & DVH-FSA & \multicolumn{2}{|c|}{$\begin{array}{c}\text { DVH-FSA vs TG-43 A } \\
\text { Diff }(\%)\end{array}$} \\
\hline Seeds & $64 \pm 7$ & $64 \pm 7$ & 0 & $64 \pm 5$ & \multicolumn{2}{|r|}{0} \\
\hline
\end{tabular}

Abbreviations: A Diff = absolute difference; DVH = dose-volume histogram; FSA = fast simulated annealing; MC = Monte Carlo; R Diff $(\%)=$ relative percent difference.

Reported are the mean and standard deviation for each DVH metric calculated using the data from all 18 patients. 

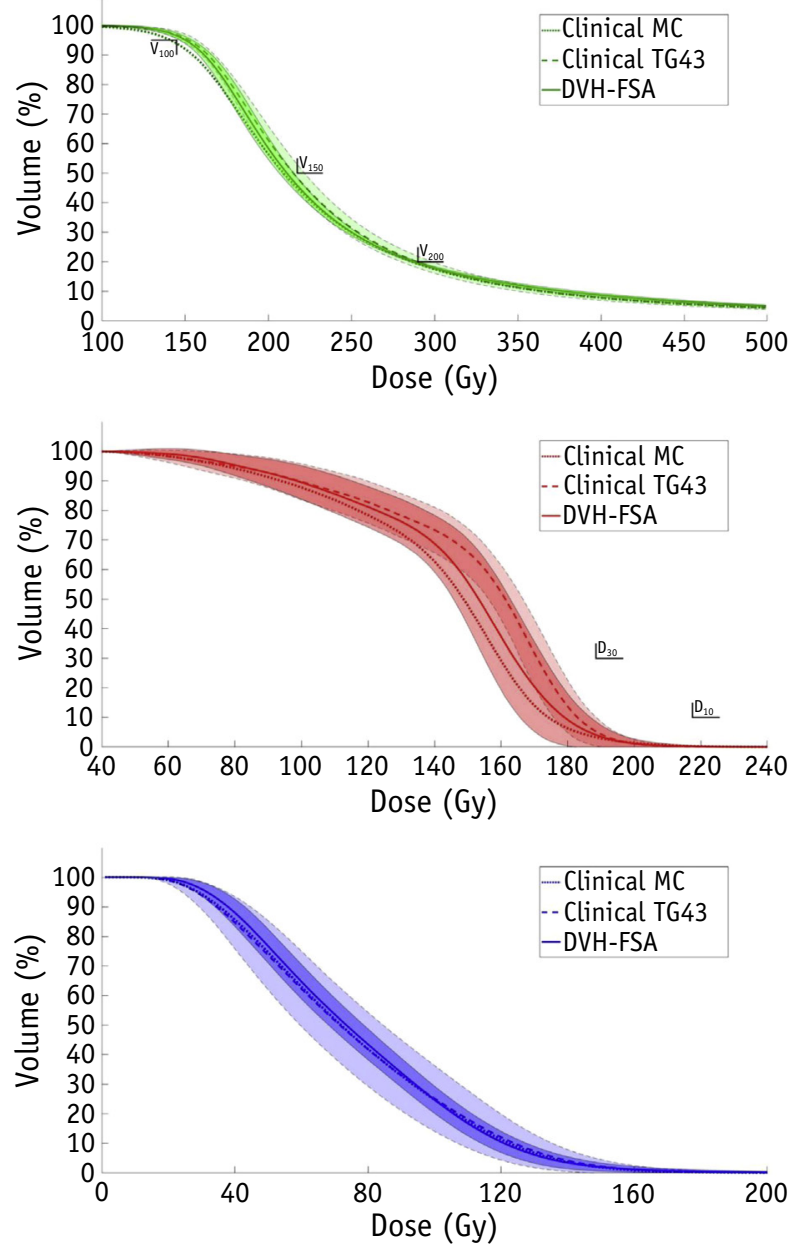

Fig. 2. Mean DVH plots of the study's population considering Clinical TG-43 (dashed), Clinical MC (dotted), and DVH-FSA (continuous) plans are given for the prostate (top), urethra (middle), and rectum (bottom). The standard deviation is given for the DVH-FSA (dark shade and continuous boundary) and the Clinical TG-43 (light shade and dashed boundary). Abbreviations: DVH $=$ dose-volume histogram; FSA $=$ fast simulated annealing; $\mathrm{MC}=$ Monte Carlo.

DVH metrics for the 18 patients considered in this study. In Figure 2, the mean DVH plots of the different scenarios and their standard deviation are compared for the prostate and critical organs. In all cases, DVH-FSA plans provided implants of good dosimetric quality with homogeneous dose coverage at the prostate while sparing the critical organs (Fig. 3).

\section{Discussion}

Previous comparisons of the AAPM TG-43 dosimetry formalism and $\mathrm{MC}$ have shown that the dose overestimation of the former can be as high as $6 \%$ for the prostate $\mathrm{D}_{100}{ }^{22}$ and up to $4 \%$ to $5 \%$ for the prostate $\mathrm{D}_{90}{ }^{20}$
In our study, the relative difference between Clinical TG-43 and Clinical MC prostate $\mathrm{D}_{90}$ was $4.3 \%$, which is in good agreement with previously reported results. Recently, a large-scale dose recalculation study for permanent implants was performed by Miksys et al. ${ }^{36}$ The authors reported lower $\mathrm{D}_{90}\left(\mathrm{~V}_{100}\right)$ in the prostate by $5.9 \% \pm 1.6 \%$ $(2.6 \pm 1.7 \%)$ and lower dose to organs at risk (OARs) of up to $6 \%$ when tissue heterogeneity and ISA effects were considered in MC simulations. Reported dose differences between Clinical TG-43 and Clinical MC plans (Table 2) were in good agreement with reported results by Miksys et al.

ISA effects, which may result in prostate $\mathrm{D}_{90}$ overestimation up to $4 \%,{ }^{21}$ were not considered in our method. However, ISA effects are not dominant for patients with intraprostatic calcifications. ${ }^{36,37}$ For these situations, the proposed DVH-FSA method may have a strong clinical impact. For patients without intraprostatic calcifications, a precalculated dose correction factor could be introduced in DVH-FSA to account for ISA effects. This correction factor may be computed with MC accounting for interseed distance and direction variations.

Moreover, DVH-FSA provided fast MC-based dosimetry $(21.5 \pm 2.5$ seconds) that complies with the intraoperative time restrictions of LDR brachytherapy. ${ }^{38}$ The planning outcome was controlled by directly optimizing the plan's DVH. Therefore, the operator's learning curve was reduced because optimization and treatment objectives were identical. Comparisons between plans calculated with the DVH-FSA technique and the clinical TG-43 formalism showed that the DVH-FSA method permitted the generation of plans with higher dose homogeneity in the prostate, with lower prostate V150 (V200) by $6.1 \%$ (5.3\%) compared with plans calculated using the TG-43 formalism. Lower dose was applied to the urethra $(4.0 \%$ lower urethra $\mathrm{D}_{10}$ ), reducing the chance for related adverse effects (eg, incontinence). The dose coverage of the prostate (prostate $\mathrm{V}_{100}$ ) satisfied the AAPM TG-137 requirements $(\geq 95 \%)$ for both DVH-FSA and Clinical TG-43 plans with negligible difference.

When tissue heterogeneity was accounted for in clinical plans (Clinical MC) the dose was reduced by up to $8.7 \%$ in the prostate and $6.8 \%$ in the OARs. Comparing the Clinical MC and DVH-FSA dosimetric results (Table 2), the former demonstrated higher dose sparing to the OARs, and the prostate $\mathrm{V}_{100}$ was lower than the TG-137 recommended value. This finding indicates that adopting AAPM TG-137 planning recommendations in MC-based planning may lead to dose escalation. This issue has been previously reported in the AAPM TG-186 report, and the necessity for MCspecific planning recommendations has been justified. Our findings provide additional justification for this necessity. Additional data on MC-based dosimetry are required to establish dose prescription guidelines dedicated to MC-based inverse planning.

The computational phantom generation from intraoperative US imaging is an efficient but less accurate 


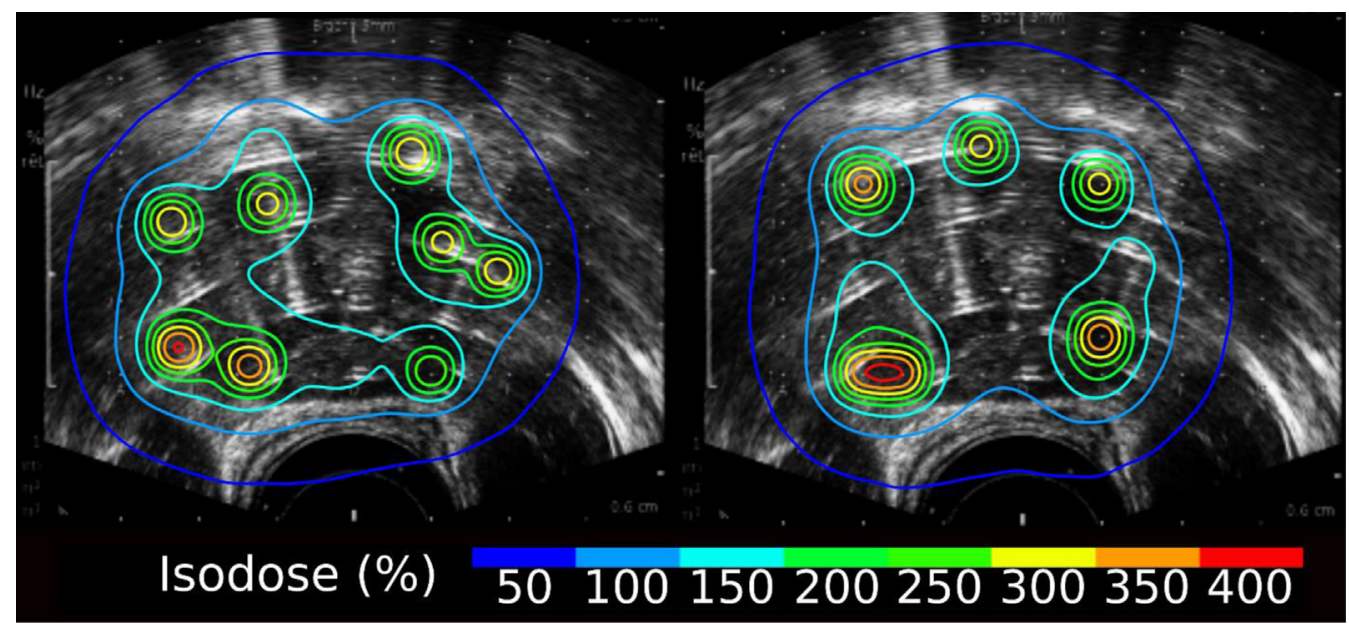

Fig. 3. Isodose distribution comparison at midgland slice between Clinical TG-43 (left) and dose-volume histogram-fast simulated annealing (right) for a representative patient.

solution compared with the CT-based voxel-by-voxel material assignment. Tissue segmentation errors resulting from low-quality ultrasound images may result in large dose errors compared with the approximation of waterequivalent medium with variable density. ${ }^{28}$ LDR brachytherapy inverse planning could capitalize on multimodal imaging and efficient image registration techniques. ${ }^{39}$ Alternatively, substitution of CT for magnetic resonance imaging-only radiation therapy ${ }^{40}$ may allow for both accurate segmentation and MC dosimetry on a voxel-byvoxel basis during LDR prostate brachytherapy inverse planning.

Another important aspect to be addressed is the postoperative alteration of the dose distribution owing to edema resorption. Postoperative dose evaluation reveals significant variations in the final dose distribution even for small edema magnitude. For instance, the intraoperative formation of edema with a magnitude between $10 \%$ and $17 \%$ can lead to a postoperative increase of the prostate $D_{90}$ from $11.7 \%$ up to $13 \% .{ }^{41,42}$ Similarly, the dose overexposure of urethra can be up to $30.0 \%$ for an edema magnitude of $27 \% \pm 9 \%{ }^{43}$ Our future objective is to consider the edemarelated postoperative dose variations within the inverse planning step. This will be addressed by introducing within the optimization process a previously developed biomechanical model for edema prediction. ${ }^{44}$ Additionally, in future work, we aim to evaluate the proposed methodology in a clinical setting. Therefore, we have developed the inverse planning software TiTAN based on the proposed DVH-FSA algorithm.

\section{Conclusions}

A DVH-FSA algorithm using GPU-accelerated MC dosimetry to account for tissue heterogeneity during LDR prostate brachytherapy inverse planning was proposed. A DVH-based cost function was minimized during optimization according to the AAPM TG-137 report's guidelines. Plans with high prostate dose homogeneity and reduced dose deposition at critical organs compared with clinical plans were generated in less than 1 minute ( $37.5 \pm 3.2$ seconds). The dose escalation risk in MC-based inverse planning with the AAPM TG-137 planning guidelines has been demonstrated. MC-specific planning guidelines should be established before adopting MC-based inverse planning in the clinical practice.

\section{References}

1. Holm HH, Juul N, Pedersen JF, et al. Transperineal 125iodine seed implantation in prostatic cancer guided by transrectal ultrasonography. J Urol 1983;130:283-286.

2. Marcus DM, Jani AB, Godette K, et al. A review of low-dose-rate prostate brachytherapy-Techniques and outcomes. J Natl Med Assoc 2010;102:500-510.

3. Mate TP, Gottesman JE, Hatton J, et al. High dose-rate afterloading 192 Iridium prostate brachytherapy: Feasibility report. Int J Radiat Oncol Biol Phys 1998;41:525-533.

4. King CR. LDR vs. HDR brachytherapy for localized prostate cancer: The view from radiobiological models. Brachytherapy 2002;1:219226.

5. Demanes DJ, Martinez AA, Ghilezan M, et al. High-dose-rate monotherapy: Safe and effective brachytherapy for patients with localized prostate cancer. Int J Radiat Oncol Biol Phys 2011;81:1286-1292.

6. Pouliot J, Tremblay D, Roy J, et al. Optimization of permanent 125I prostate implants using fast simulated annealing. Int J Radiat Oncol Biol Phys 1996;36:711-720.

7. Yu Y, Schell MC. A genetic algorithm for the optimization of prostate implants. Med Phys 1996;23:2085-2091.

8. D’Amours M, Pouliot J, Dagnault A, et al. Patient-specific Monte Carlo-based dose-kernel approach for inverse planning in afterloading brachytherapy. Int J Radiat Oncol Biol Phys 2011;81:1582-1589.

9. Citrin D, Ning H, Guion P, et al. Inverse treatment planning based on MRI for HDR prostate brachytherapy. Int J Radiat Oncol Biol Phys 2005;61:1267-1275.

10. Pouliot J, Kim Y, Lessard E, et al. Inverse planning for HDR prostate brachytherapy used to boost dominant intraprostatic lesions defined by magnetic resonance spectroscopy imaging. Int J Radiat Oncol Biol Phys 2004;59:1196-1207. 
11. Lessard E, Pouliot J. Inverse planning anatomy-based dose optimization for HDR-brachytherapy of the prostate using fast simulated annealing algorithm and dedicated objective function. Med Phys 2001; 28:773-779.

12. Guthier C, Aschenbrenner KP, Buergy D, et al. A new optimization method using a compressed sensing inspired solver for real-time LDR-brachytherapy treatment planning. Phys Med Biol 2015;60: 2179-2194.

13. Li Z, Liu C, Palta JR. Optimized dose distribution of a high dose rate vaginal cylinder. Int J Radiat Oncol Biol Phys 1998;41:239244.

14. Nath R, Bice WS, Butler WM, et al. AAPM recommendations on dose prescription and reporting methods for permanent interstitial brachytherapy for prostate cancer: Report of Task Group 137. Med Phys 2009;36:5310-5322.

15. Cotrutz C, Xing L. Using voxel-dependent importance factors for interactive DVH-based dose optimization. Phys Med Biol 2002;47: 1659-1669.

16. Spirou SV, Chui CS. A gradient inverse planning algorithm with dosevolume constraints. Med Phys 1998;25:321-333.

17. Kirisits C, Pötter R, Lang S, et al. Dose and volume parameters for MRI-based treatment planning in intracavitary brachytherapy for cervical cancer. Int J Radiat Oncol Biol Phys 2005;62:901-911.

18. Chen Y, Boyer AL, Xing L. A dose-volume histogram based optimization algorithm for ultrasound guided prostate implants. Med Phys 2000;27:2286-2292.

19. Nath R, Anderson LL, Luxton G, et al. Dosimetry of interstitial brachytherapy sources: Recommendations of the AAPM Radiation Therapy Committee Task Group No. 43. Med Phys 1995;22:209-234.

20. Carrier JF, Beaulieu L, Therriault-Proulx F, et al. Impact of interseed attenuation and tissue composition for permanent prostate implants. Med Phys 2006;33:595-604.

21. Carrier JF, D’Amours M, Verhaegen F, et al. Postimplant dosimetry using a Monte Carlo dose calculation engine: A new clinical standard. Int J Radiat Oncol Biol Phys 2007;68:1190-1198.

22. Chibani O, Williamson JF, Todor D. Dosimetric effects of seed anisotropy and interseed attenuation for Pd103 and I125 prostate implants. Med Phys 2005;32:2557-2566.

23. Bush K, Gagne IM, Zavgorodni S, et al. Dosimetric validation of Acuros XB with Monte Carlo methods for photon dose calculations. Med Phys 2011;38:2208-2221.

24. Sempau J, Wilderman SJ, Bielajew AF. DPM, a fast, accurate Monte Carlo code optimized for photon and electron radiotherapy treatment planning dose calculations. Phys Med Biol 2000;45:2263-2291.

25. Hissoiny S, Ozell B, Bouchard H, et al. GPUMCD: A new GPUoriented Monte Carlo dose calculation platform. Med Phys 2011;38: 754-764.

26. Lemaréchal Y, Bert J, Falconnet C, et al. GGEMS-Brachy: GPU GEant4-based Monte Carlo simulation for brachytherapy applications. Phys Med Biol 2015;60:4987-5006.

27. Bert J, Perez-Ponce H, El Bitar Z, et al. Geant4-based Monte Carlo simulations on GPU for medical applications. Phys Med Biol 2013;58: 5593-5611.
28. Beaulieu L, Carlsson Tedgren $\AA$, Carrier JF, et al. Report of the Task Group 186 on model-based dose calculation methods in brachytherapy beyond the TG-43 formalism: Current status and recommendations for clinical implementation. Med Phys 2012;39:6208-6236.

29. Hsieh J. Adaptive streak artifact reduction in computed tomography resulting from excessive x-ray photon noise. Med Phys 1998;25:21392147.

30. Landry G, Granton PV, Reniers B, et al. Simulation study on potential accuracy gains from dual energy CT tissue segmentation for lowenergy brachytherapy Monte Carlo dose calculations. Phys Med Biol 2011;56:6257-6278.

31. Bethesda M. Photon, electron, proton and neutron interaction data for body tissues. ICRU Report 46 1992;46:13.

32. Valentin J. Basic anatomic and physiological data for use in radiologic protection: Reference values: ICRP Publication 89. Ann ICRP 2002; $32: 1-277$.

33. Williamson JF. Monte Carlo evaluation of kerma at a point for photon transport problems. Med Phys 1987;14:567-576.

34. Bonenfant É, Magnoux V, Hissoiny S, et al. Fast GPU-based Monte Carlo simulations for LDR prostate brachytherapy. Phys Med Biol 2015;60:4973-4986.

35. Kirov AS, Williamson JF. Monte Carlo-aided dosimetry of the Source Tech Medical Model STM1251 I-125 interstitial brachytherapy source. Med Phys 2001;28:764-772.

36. Miksys N, Vigneault E, Martin AG, et al. Large-scale retrospective Monte Carlo dosimetric study for permanent implant prostate brachytherapy. Int J Radiat Oncol Biol Phys 2017;97:606-615.

37. Collins Fekete CA, Plamondon M, Martin AG, et al. Calcifications in low-dose rate prostate seed brachytherapy treatment: Postplanning dosimetry and predictive factors. Radiother Oncol 2015;114:339-344.

38. Beyer DC, Shapiro RH, Puente F. Real-time optimized intraoperative dosimetry for prostate brachytherapy: A pilot study. Int J Radiat Oncol Biol Phys 2000;48:1583-1589.

39. Hamdan I, Bert J, Rest CCL, et al. Fully automatic deformable registration of pretreatment MRI/CT for image guided prostate radiotherapy planning. Med Phys 2017;44:6447-6455.

40. Edmund JM, Nyholm T. A review of substitute CT generation for MRI-only radiation therapy. Radiat Oncol 2017;12:28.

41. Waterman FM, Yue N, Corn BW, et al. Edema associated with I-125 or Pd-103 prostate brachytherapy and its impact on postimplant dosimetry: An analysis based on serial CT acquisition. Int J Radiat Oncol Biol Phys 1998:41:1069-1077.

42. Chira C, Delouya G, Larrivée S, et al. Prostate volume changes during permanent seed brachytherapy: An analysis of intraoperative variations, predictive factors and clinical implication. Radiat Oncol 2013;8: 177.

43. Waterman FM, Dicker AP. The impact of postimplant edema on the urethral dose in prostate brachytherapy. Int J Radiat Oncol Biol Phys 2000;47:661-664.

44. Mountris KA, Bert J, Noailly J, et al. Modeling the impact of prostate edema on LDR brachytherapy: A Monte Carlo dosimetry study based on a 3D biphasic finite element biomechanical model. Phys Med Biol 2017;62:2087-2102. 